\title{
Minimum Volume for a Liquid Bridge between Equal Disks
}

\author{
ANGEL SANZ AND ISIDORO MARTINEZ \\ Polytechnic University of Madrid, Spain
}

Received April 20, 1982; accepted September 9, 1982

\begin{abstract}
The lower stability limit for axisymmetric floating zones at rest between equal coaxial disks has been experimentally verified for several disk-separation/disk-diameter ratios by using the neutral buoyancy technique. Results show a close agreement with theory in the case of bridge disruption and a wide scatter in the case of edge detachment.
\end{abstract}

\section{INTRODUCTION}

This work tries to fill a gap found in available experimental data on the stability of floating zones at rest; i.e., the volume of breakage of a liquid bridge held between parallel, concentric, equal disks under the sole action of interfacial forces.

Theoretical predictions were made by Gillette and Dyson (1) and by Da Riva and Martínez (2) after which the diagram shown in Fig. 1 has been prepared. The limit due to detachment from the disk edge corresponds to curve $0 A$ for zero contact angle; curve $A B$ corresponds to a local minimum in liquid volume, and curve $B C$ to nonsymmetric rupture (but still axisymmetric) by bifurcation to unstable shapes. The upper bound (curve $0 D$ ) is arbitrarily taken to correspond to a contact angle of $180^{\circ}$ at the edge, but has no relevance to this study.

Figure 2 shows the theoretical limit for detachment for several other contact angles, which have been computed after (2) in an attempt to clarify the wide scatter found in these trials.

Gravity effects may be kept out of the analysis when its ratio to surface tension effects, measured by the Bond number $B=\Delta \rho g L D /$ $\sigma$, is much smaller than unity, $\Delta \rho$ being the density difference between the liquid zone and the surrounding fluid, $g$ the local gravity level, $L$ a characteristic vertical length, $D$ a characteristic radius of curvature for the interface, and $\sigma$ the surface tension.

The different approaches to achieve $B$ $\ll 1$ characterize different experimental techniques: neutral buoyancy (Plateau simulation) makes $\Delta \rho \ll 1$ by surrounding the liquid zone with another immiscible liquid of the same density. However, dynamical effects are masked and some doubts are cast on the relevance of the wetting conditions, though here the three-phase line is anchored at the edge of the disk. Other experimental techniques are even less convenient: soap films give a negligible $\Delta \rho$ but present hard handling problems, and the decreasing of dimensions ( $L$ and $D$ ) to get $B \ll 1$ without altering either $\Delta \rho$ or $g$ demands sophisticated experimental means to control and measure minute amounts of liquid (of the order of some cubic millimeters). Changing $\sigma$ is not a solution because recourse would have to be taken to mercury or molten metals, and problems would increase. Of course, the easiest way out is to have access to microgravity conditions using short-term parabolic flights of specially equipped airplanes or sounding rockets, or a long-term but much more expensive orbiting platform such as Spacelab. This last approach is the only one suited to some application research where large samples are required. 


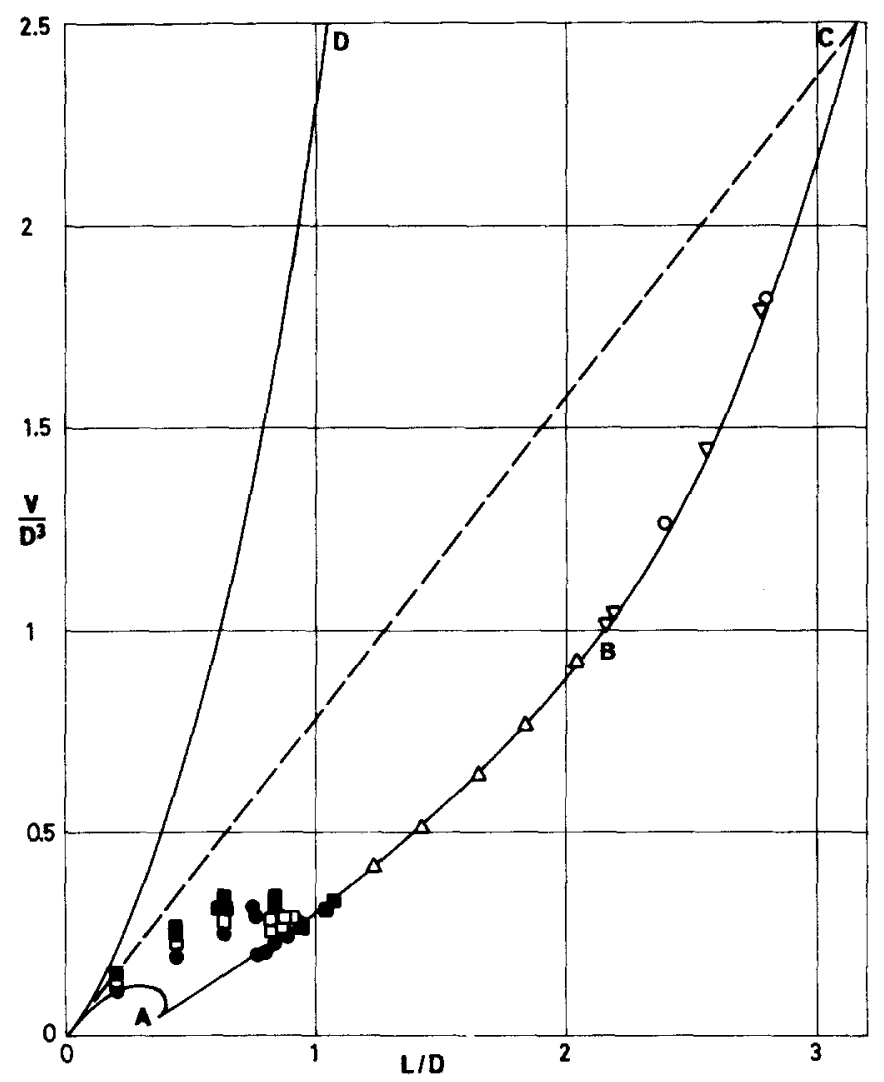

FIG. 1. Minimum volume $V$ for a liquid floating zone of length $L$, held by surface tension between two equal disks of diameter $D$. Solid curves correspond to theoretical predictions (2). The dashed curve corresponds to cylindrical shapes. Equal signed points belong to the same set of trials in this experiment.

The published experimental work on the stability of floating zones may be grouped as follows: Mason (3) studied the stability of cylindrical zones at rest in neutral buoyancy; Carruthers and Grasso (4) added the influence of gravity and rotation to the stability of cylindrical and conical zones vertically placed in a plateau tank, and that of rotation (still in the cylindrical case) in a microgravity environment (5); Coriell et al. (6) considered cylindrical zones oriented both vertically and horizontally in a plateau tank, and also carried out tests with microzones; Fowle et al. (7) also used this technique to study cylindrical and conical zones with and without solid-body rotation. Incidentally, only Coriell et al. present data on maximum length versus volume in the neighborhood of the cylindrical breakage, i.e., for $L / D=\pi$ ( $L$ being the length and $D$ the diameter of the disk) and only for $B=1.6$.

\section{MATERIALS}

Experiments were carried out in a Plateau Tank Facility (PTF), described in Martínez and Rivas (8), specially developed to simulate the Fluid Physics Module (FPM) to be flown on Spacelab. The relevant PTF capabilities as used in this set of trials can be seen sketched in Fig. 3. The floating zone is established by injecting some liquid to form a cap on the upper disk and then touching the lower one with the cap to force the merging. Good spreading and anchoring conditions are assured by the high mobility of the upper 
disk, the lower one being fixed to the tank floor. Wetting conditions are controlled through surface treatment of the disk lateral faces with an antispreading barrier (FC-721 from $3 \mathrm{M}$ ). Two sets of perspex disks were used: a 30-mm-diam set for long zones and a 50-mm one for short zones.

The tank is a glass box with a square cross section $100 \mathrm{~mm}$ on a side and $130 \mathrm{~mm}$ in height. Liquid for the bath (a 1:2 mixture of water-methanol) was filled via a siphon to avoid bubble formation. Liquid for the floating zone (a dimethyl-silicone oil with a viscosity 20 times that of water) was injected by gravity using a graduated burette with a capacity of $100 \mathrm{~cm}^{3}$ and an accuracy of \pm 0.1 $\mathrm{cm}^{3}$. To enhance the visualization of the interface, background illumination was obtained by placing a $60-\mathrm{W}$ lamp $30 \mathrm{~cm}$ behind the rear glass panel on which a translucent grid was attached. The difference in refractive index between the liquid of the zone and that of the outer bath gave rise to a magnifyingglass effect that greatly improved the visualization of interfacial distortions (see Fig. 4). A video camera, placed $1.5 \mathrm{~m}$ away to $\mathrm{min}$ imize conical effects on viewing, was used to

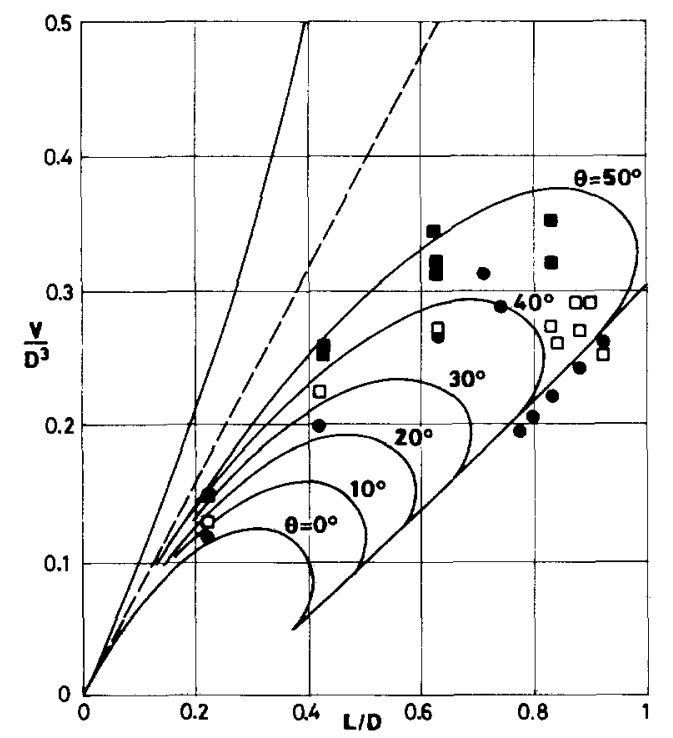

FIG. 2. Enlargement of lower part of Fig. 1 showing the wide scatter for short zones $(L / D<1)$.

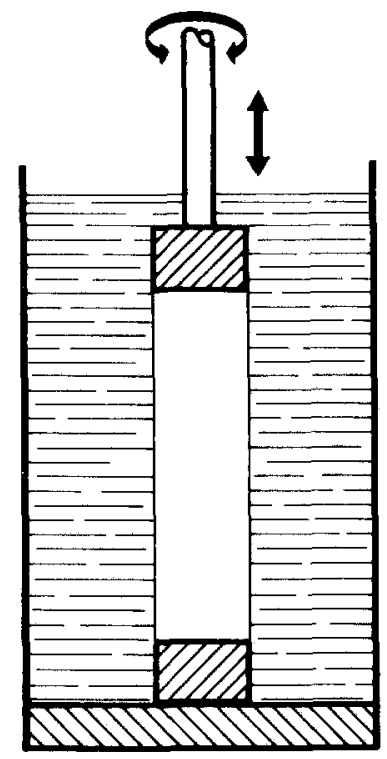

FIG. 3. Sketch of the experimental setup.

present an enlarged image in a monitor and record selected sequencies for detailed analysis.

The density of both the mixture and the working liquid was measured to be $\rho=954$ $\pm 0.5 \mathrm{~kg} \mathrm{~m}^{-3}$, but the residual imbalance limited the maximum achievable disk separation to $L / D \sim 2$ just after this initial stage. Thus a fine density matching was performed before every experiment by adding either alcohol or water to the bath. To this purpose, the zone itself was used as the best density indicator, accuracy being gained as the $L / D$ ratio approached the stability limit, though slowness and extreme care in handling were needed (with $L / D=3.1$ uncontrolled disturbances caused disruption after some minutes). The floating-zone volume in this density matching was always kept equal to that of a cylindrical bridge, $V=\pi D^{2} L / 4$, and the magnifying-glass effect used to detect variations of the cylindrical shape (details are given in the Appendix).

Alcohol additions of the order of $1 \mathrm{~cm}^{3}$ should cause a density variation $\Delta \rho / \rho \sim 10^{-4}$ which is sufficient to produce a large zone distortion according to the estimates made in the Appendix; however, no significant 


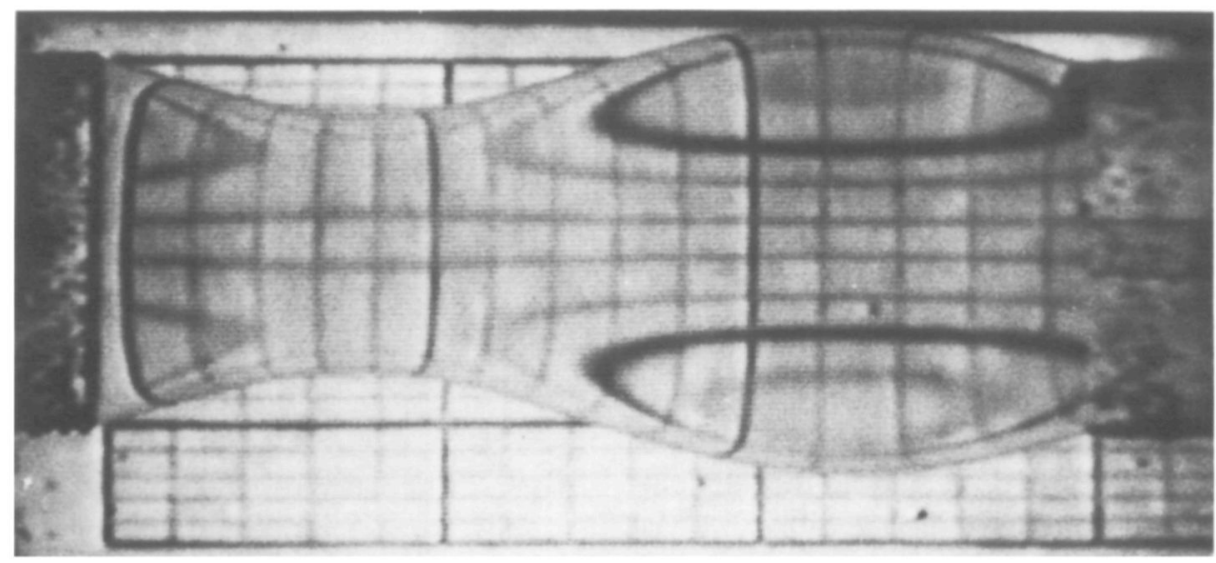

FIG. 4. Photograph showing the magnifying-glass effect of the zone.

changes in shape appear in practice when such minute quantities are added, at least in a reasonable time, because of the difficulty in homogenizing the mixture.

\section{METHODS AND RESULTS}

Before every experiment, a fine density matching was performed to compensate for the loss of alcohol due to evaporation and for temperature variations which, although only of the order of $0.1^{\circ} \mathrm{C}$ during the trial, amounted to $1^{\circ} \mathrm{C}$ between trials (because of the long preparation period). The density matching was performed by setting up a cylindrical zone of $L / D=2.8$ for breakage trials with $L / D<2.5$, and going up to $L / D=3$ for breakages up to $L / D=2.8$ that was the highest ratio tried. Above this $L / D$ ratio the setting time was so long that density gradients took place in parallel with velocity damping, and the preparation was tedious and unreliable.

Trials can be divided in two groups: those for $L / D>1$, where disruption of the liquid bridge took place, and those for $L / D<1$, where the liquid just detached from the disk edge.

For slender zones, $L / D>1$, disks of 30 $\mathrm{mm}$ diameter were used. After the density matching using a longer cylindrical column, the desired $L / D$ ratio and appropriate liquid volume were set. The experiment consists in sucking liquid from the zone at constant $L$ until the bridge disrupts. From the cylindrical configuration a quick withdrawal (at 0.3 $\mathrm{cm}^{3} \mathrm{sec}^{-1}$ ) is performed to bring the zone to the vecinity of the theoretical limit of minimum volume. This is followed by a slow withdrawal in steps of $0.2 \mathrm{~cm}^{3}$ (at $0.1 \mathrm{~cm}^{3}$ $\mathrm{sec}^{-1}$ ) waiting some minutes for settling in between to allow for instabilities, if any, to develop. The accuracy in volume and $L / D$ ratio measurements was $1 \%$. Results are shown in Fig. 1. The remarkable fit to the theoretical predictions greatly simplified the trials because breakage was expected to occur at such a well defined volume.

Results also show the different disruption character for zones longer and shorter than $L / D=2.13$. For lower $L / D$ ratios the shape necks at the middle and the neck remains at the mid-plane between disks during the whole breakage sequence. For $L / D>2.13$ the neck also starts at the mid-plane but, once the stability limit is reached, it shifts axially toward one of the disks at nearly constant neck diameter, and the necking then proceeds until total disruption occurs. In both cases, apart from the two liquid caps attached to the disks (equal in size for $L / D<2.13$ and different for $L / D>2.13$ ) a small satellite drop, the size of which increases with $L / D$, is formed from the liquid filament that joins the caps just before the rupture (Fig. 5). 
For $L / D$ well below unity the detachment from the disk edge takes precedence over the bridge disruption. To better monitor this detachment a larger 50-mm-diam disk set was used. Even so, results showed much wider scatter than for bridge rupture; the threephase line formed between the oil/water-alcohol mixture over the perspex disk did not show a constant contact angle (see Fig. 2) perhaps due to interface inhomogeneities, contamination, edge scratches, and so on. Because of these difficulties, a slow liquid withdrawal was started directly from the cylindrical configuration unlike the $L / D>1$ runs. The number of runs was also increased to have some statistical average and a mean contact angle of some $40^{\circ}$ was found (see Fig. 2).

These points in Fig. 2 which fall under the theoretical limit must be taken with caution because they correspond to liquid bridge disruption after a detachment not detected in time and the given limits are not applicable.

With the 50-mm disks, the density matching was a problem since no $L / D>2$ zones could be obtained in the test rig. To overcome this drawback, these large disks were machined with an inner protrusion: a working surface of $30-\mathrm{mm}$ diameter protruding $1 \mathrm{~mm}$, by means of which the density matching was accomplished as stated above for $L /$ $D>1$, adding more liquid afterwards to overflow the 1-mm jump and spread toward the $50-\mathrm{mm}$ edges. In any case, because of the relatively small heights needed for the $L / D$ $<1$ trials, density balance was not so critical.

\section{CONCLUSIONS}

The lower stability limit for floating zones between equal coaxial disks has been experimentally verified for several $L / D$ ratios in a neutral buoyancy apparatus. Results show extremely close agreement with theory in the case of bridge disruption $(L / D>1)$ but a wide scattering takes place in the case of edge detachment, showing a poorly defined limiting contact angle near $40^{\circ}$.

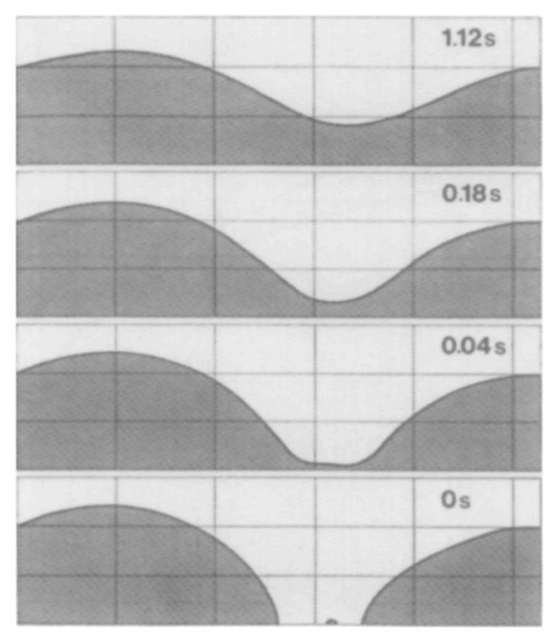

FIG. 5. Sketch of the breakage sequence of a zone with $L / D=2.7$ taken from the videotape recording. Numbers show time to the bridge disruption.

The main experiment problem faced has been the difficult density matching arising from the tendency of the water-alcohol mixture used for the outer bath to extratify and evaporate.

\section{APPENDIX}

Shape Distortion of a Cylindrical Zone Due to Residual Axial Gravity

Using $D$ as unit length, the undistorted cylinder is $r(z)=1 / 2$ for $0 \leqslant z \leqslant \Lambda$, where $\Lambda=L / D$. The distorted shape will be

$$
r(z)=1 / 2[1+B f(z)],
$$

where $B=\Delta \rho g L D / \sigma$ stands for a nonzero Bond number which is the cause of the disturbance, and $f(z)$ is to be found after the local balance of pressure at the interface, which in nondimensional terms takes the form

$$
\begin{aligned}
r^{\prime \prime}\left(1+r^{\prime 2}\right)^{-3 / 2}-r^{-1}(1 & \left.+r^{\prime 2}\right)^{-1 / 2} \\
& +B z / \Lambda+p=0
\end{aligned}
$$

$p$ being the pressure jump at a reference level, an internal unknown to add to the problem.

The boundary conditions are 


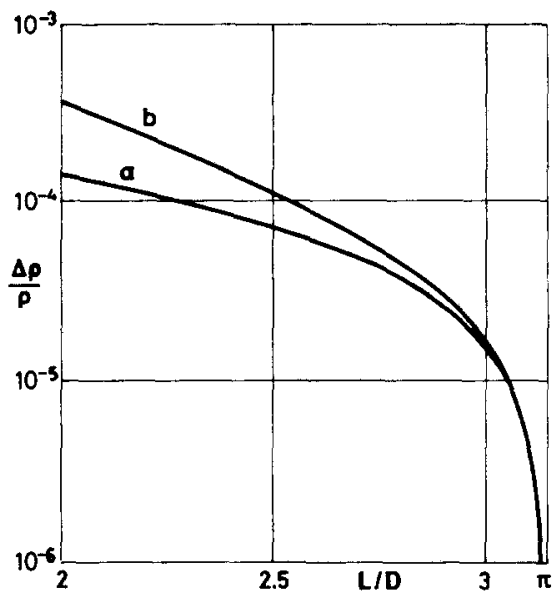

FIG. 6. Theoretical density matching required $\Delta \rho / \rho$ (corresponds to the microgravity level) versus $L / D$ ratio for a cylindrical zone to deviate $\sim 3 \%$ ( $1 \mathrm{~mm}$ in the 30 mm-diam disks used) due to the residual density imbalance: (a) from Eq. [5], (b) from Eq. [6].

$$
\begin{gathered}
r(0)=r(\Lambda)=1 / 2 \\
\pi \int_{0}^{\Lambda} r^{2} d z=\pi \Lambda / 4
\end{gathered}
$$

Thus, the linear solution stated in [1] takes the form

$$
\begin{aligned}
r=\frac{1}{2}\left[1+\frac{B}{4}\left(\frac{\Lambda-2 z}{\Lambda}\right.\right. & \\
& \left.\left.-\frac{\sin (\Lambda-2 z)}{\sin \Lambda}\right)\right] .
\end{aligned}
$$

From [4] it can be easily seen that the distortion diverges as $\Lambda$ approaches $\pi$. The peakto-peak amplitude is

$$
\begin{aligned}
r_{\max }-r_{\min }= & \frac{B}{4 \Lambda}\left(\sqrt{\frac{\Lambda^{2}}{\sin ^{2} \Lambda}-1}\right. \\
& \left.-\arctan \sqrt{\frac{\Lambda^{2}}{\sin ^{2} \Lambda}-1}\right)
\end{aligned}
$$

which, for long zones $(\Lambda \sim 3)$ can be simplified to

$$
r_{\max }-r_{\min }=\frac{B}{4 \sin \Lambda} .
$$

Typical experimental values are $D=30$ $\mathrm{mm}, \Lambda=3, r_{\max }-r_{\min }=0.03, g=10 \mathrm{~m}$ $\mathrm{sec}^{-2}, \rho=10^{3} \mathrm{~kg} \mathrm{~m}^{-3}$, and $\sigma=0.02 \mathrm{~N} \mathrm{~m}^{-1}$, for which a relative density matching

$$
\Delta \rho / \rho=4 \sin \Lambda\left(r_{\max }-r_{\min }\right)(\sigma / \rho g L D)
$$

in the order of $10^{-5}$ is required, clearly showing the extreme care demanded.

Figure 6 gives an idea of the relative density matching required, which corresponds exactly to the microgravity level attained, to have a liquid column of cylindrical volume, $V=\pi D^{2} L / 4$, with a maximum distortion from cylindrical shape of $1 \mathrm{~mm}$, as a function of $\Lambda$. The value used for the interfacial tension $\sigma$ for the couple water-alcohol/siliconeoil, is just an estimate.

\section{ACKNOWLEDGMENT}

The authors acknowledge the support of their research by grants from the Spanish Space Research Commission (CONIE).

\section{REFERENCES}

1. Gillette, R. D., and Dyson, D. C., Chem. Eng. $J$. 2, 44 (1971).

2. Da Riva, I., and Martínez, I., ESA SP-142, 67 (1979).

3. Mason, G., J. Colloid Interface Sci. 32, 172 (1970).

4. Carruthers, J. R., and Grasso, M., J. Appl. Phys. 43, 436 (1972).

5. Carruthers, J. R., Gibson, E. G., and Klett, M. G., Progr. Astronaut. Aeronaut. 52, 207 (1977).

6. Coriell, S. R., Hardy, S. C., and Cordes, M. R., J. Colloid Interface Sci. 60, 126 (1977).

7. Fowle, A. A., et al., ESA SP-142, 317 (1979).

8. Martínez, I., and Rivas, D., Acta Astronautica 9, 339 (1982). 